\title{
Phase locking between excess spike synchrony and LFP is independent of rate covariation
}

\author{
Michael Denker ${ }^{1 *}$, Alexa Riehle ${ }^{2}$, Markus Diesmann ${ }^{1,3}$, Sonja Grün ${ }^{1}$ \\ From Nineteenth Annual Computational Neuroscience Meeting: CNS*2010 \\ San Antonio, TX, USA. 24-30 July 2010
}

A possible strategy of information coding employed by cortical networks involves the propagation of activity through synchronously firing groups of neurons, termed assemblies. A hallmark signature of such an assembly coding scheme is the detection of synchronous spiking in parallel recordings of neuronal activity. Experimental work (e.g., [1]) indirectly substantiates the assembly idea with findings of behavioral correlates of significant excess synchronous spiking activity. Recently, we demonstrated [2] that such excess coincident spikes, or Unitary Events [3], also show an exceptionally strong phase-locking to oscillations of the local field potential (LFP), a signal measured directly at the population level. A theoretical model consistently embeds this experimentally observed relationship within the assembly framework. In this context, the weak entrainment of spikes of a single neuron to the LFP is explained as a reflection of the neuron's participation in different assemblies across the network.

Co-varying firing rate modulations induced by LFP oscillations were instead suggested as the cause for preferred locking of excess spike coincidences to the LFP phase. In this study we investigate if this alternative hypothesis can be validated. To this end, we first identify the extremal rate changes within those UE analysis windows that are assumed to be expressions of a rate step. We then relate the magnitude of individual rate steps to the degree of rate co-variation across neurons. In a final step, we relate these two measures to phase and amplitude of the LFP. We find that the locking of significant spike synchrony does not preferentially occur with rate changes.

As an additional test we extended our previous work by replacing the parametric distribution of coincidences used for evaluating the significance of observed coincidence counts by numerically derived distributions based

\footnotetext{
* Correspondence: mdenker@brain.riken.jp

${ }^{1}$ RIKEN Brain Science Institute, Wako City, Japan
}

on surrogate data. The employed surrogate method (spike train dithering, see [4]) considers the statistical features (non-stationarity of rates, interspike interval distribution,...) of the experimental spike trains, while destroying precise spike coincidences. Simulations show that this method is conservative compared to the UE analysis used in earlier work. Despite the decreased sensitivity of the surrogate based method, this additional analysis confirms our previous findings on the relationship between spike synchrony and the LFP, and demonstrates that they are not explained as a consequence of rate covariation. Taken together, these two analyses corroborate our original interpretation of LFP oscillations as a reflection of assembly dynamics.

\section{Acknowledgements}

Partially funded by the Helmholtz Alliance on Systems Biology, the French National Research Agency (ANR-05-NEUR-045-01), and the Next-Generation Supercomputer Project of MEXT (Japan).

\section{Author details}

${ }^{1}$ RIKEN Brain Science Institute, Wako City, Japan. ${ }^{2}$ INCM, CNRS - Univ. Aix-Marseille 2, Marseille, France. ${ }^{3}$ Brain and Neural Systems Team, RIKEN Computational Science Research. Program, Wako City, Saitama, Japan.

Published: 20 July 2010

\section{References}

1. Riehle A, Grün S, Diesmann M, Aertsen A: Spike synchronization and rate modulation differentially involved in motor cortical function. Science 1997, 278:1950-1953.

2. Denker $M$, Roux $S$, Lindén $H$, Diesmann $M$, Riehle $A$, Grün S: Reflections of synchronous spiking activity in local field potentials. Online Abstract Viewer/tinerary Planner. San Diego, CA. Society for Neuroscience 2007, Program No. 793.4.

3. Grün $S$, Diesmann $M$, Aertsen A: Unitary events in multiple single-neuron spiking activity: II. Nonstationary data. Neural Comput 2002, 14:81-119.

4. Grün S: Data-driven significance estimation for precise spike correlation. J Neurophysiol 2009, 101:1126-1140.

doi:10.1186/1471-2202-11-S1-P4

Cite this article as: Denker et al:: Phase locking between excess spike synchrony and LFP is independent of rate covariation. BMC Neuroscience 2010 11(Suppl 1):P4. 